\title{
BMJ Open Exploring the personal burden of shoulder pain among younger people in Australia: protocol for a multicentre cohort study
}

\author{
Ilana N Ackerman, ${ }^{1}$ Richard S Page, ${ }^{2}$ Kathy Fotis, ${ }^{1}$ Peter Schoch, ${ }^{3}$ \\ Nigel Broughton, ${ }^{4,5}$ Sharon L Brennan-Olsen, ${ }^{6,7}$ Andrew Bucknill, ${ }^{8}$ Emily Cross ${ }^{9}$
}

To cite: Ackerman IN, Page RS, Fotis K, et al. Exploring the personal burden of shoulder pain among younger people in Australia: protocol for a multicentre cohort study. BMJ Open 2018;8:e021859. doi:10.1136/ bmjopen-2018-021859

- Prepublication history for this paper is available online. To view these files, please visit the journal online (http://dx.doi. org/10.1136/bmjopen-2018021859).

INA and RSP contributed equally.

Received 30 January 2018 Revised 5 May 2018

Accepted 12 June 2018

\section{D) Check for updates}

(C) Author(s) (or their employer(s)) 2018. Re-use permitted under CC BY-NC. No commercial re-use. See rights and permissions. Published by BMJ.

For numbered affiliations see end of article.

\section{Correspondence to} Associate Professor llana N Ackerman;

ilana.ackerman@monash.edu

\section{ABSTRACT}

Introduction Persistent musculoskeletal conditions can impact profoundly on younger people's quality of life, psychological distress and capacity to work, as shown by previous research involving younger people with osteoarthritis. The personal impacts, in particular, work and parenting impacts, of other musculoskeletal conditions (such as persistent shoulder pain) on younger patient groups remain poorly understood. Furthermore, the personal financial burden associated with managing musculoskeletal conditions is rarely documented. This study aims to investigate well-being, work participation and productivity, shoulder-related parenting disability and out-of-pocket healthcare expenditure among younger people with shoulder pain and evaluate changes over 12 months.

Methods and analysis One hundred and fifty people aged 20-55 years with shoulder pain of more than 6 weeks' duration (excluding those with recent history of fracture or dislocation) will be recruited for this cohort study. Participants will be recruited from three major public hospitals in Victoria, Australia, following screening of orthopaedic outpatient clinics lists and referrals. Participants will be asked to complete a baseline questionnaire and 2-week healthcare costs diary, with follow-up data collected at 12 months. Patient-reported outcomes will be collected, including health-related quality of life (HRQoL), shoulder pain and function, psychological distress, shoulder-related parenting disability and work productivity. Information on sociodemographics, employment, health services utilisation and shoulderrelated healthcare expenditure will also be collected. Descriptive analysis of baseline data will provide a comprehensive snapshot of the personal burden of shoulder pain. Baseline HRQoL and psychological distress data will be compared with Australian population norms to provide context around well-being. Associations between sociodemographic factors and patient-reported outcomes will be evaluated using univariate and multivariate analyses. Changes in patient-reported outcomes from baseline to 12 months will be analysed using paired t-tests.

Ethics and dissemination Ethics approval has been obtained. The study findings will be submitted to peerreviewed journals and presented at relevant scientific meetings.

\section{Strengths and limitations of this study}

- A key strength of this study is the breadth of outcomes relevant to younger people that will be captured (including health-related, work-related and parenting-related impacts plus out-of-pocket healthcare costs).

- The recruitment strategy involves metropolitan and regional public hospitals to improve external validity.

- Patients seen only in primary care settings will not be included in the study; however, individuals with ongoing shoulder pain would be referred for specialist opinion and management from a primary healthcare setting.

- An exploration of longer term impacts (beyond 12 months) would be valuable but is not currently feasible within available research funding.

\section{INTRODUCTION}

Persistent musculoskeletal conditions can impact profoundly on younger people, in terms of impaired quality of life, increased psychological distress and reduced capacity to work and maintain employment. This was clearly demonstrated in our earlier research involving younger people with hip and knee osteoarthritis. ${ }^{1}$ The impacts of other common painful musculoskeletal conditions (such as shoulder pain) on younger patient groups remain poorly understood, particularly with regard to the potential work and parenting impacts. Furthermore, the personal financial burden associated with managing musculoskeletal conditions is rarely documented. Direct healthcare costs borne by the patient are likely to encompass medical and surgical consultations, visits to physiotherapists and other allied health practitioners, as well as the costs of diagnostic tests and medications. A subgroup of patients with unresolved pathology also bear the costs of surgery and associated rehabilitation and time off work. 
Shoulder symptoms are relatively common in the community, affecting $22 \%$ of adults in a large population-based survey from South Australia. ${ }^{2}$ A systematic review of general population studies reported that the point prevalence of shoulder pain in the general population aged less than 70 years ranged from $7 \%$ to $27 \%$, while the lifetime prevalence of shoulder pain was up to $67 \% .^{3}$ While shoulder pain can occur across the lifespan, people of working age are commonly affected given exposures to occupational risk factors ${ }^{45}$ and risk of injury from recreational or competitive sport participation. ${ }^{67}$ To date, a number of observational studies have evaluated constructs such as quality of life, mental health, general health status and shoulder function in people with painful shoulder conditions; however, the mean age of participants was over 50 years. ${ }^{8-12}$ An early study of common shoulder conditions (such as glenohumeral instability, osteoarthritis and adhesive capsulitis) did involve younger participants (with an average age of 31 years for the instability subgroup), but only collected limited outcomes data using the SF-36 Health Survey. ${ }^{13}$ While age-specific pain and physical function scores have been reported for subgroups of younger people in the general community with shoulder pain, ${ }^{2}$ the broader impacts of shoulder pain on other aspects of well-being remain unclear. Additionally, some studies have evaluated the impacts of shoulder pain combined with other bodily pain (eg, neck/shoulder pain ${ }^{14}$ ), making the specific contribution of shoulder pain impossible to elucidate.

Improving our understanding of the personal, work-related and financial impacts of painful shoulder conditions is essential for optimising patient care and will assist in planning future health services and supportive programmes to meet the needs of this patient group. The overarching aim of this study is to investigate well-being, work participation, work productivity and out-of-pocket healthcare expenditure among younger people with shoulder pain presenting to public orthopaedic outpatient clinics. The specific aims are to:

1. Compare the health-related quality of life (HRQoL) of people aged between 20 years and 55 years who have shoulder pain with age-matched and sex-matched Australian population norms (primary aim).

2. Evaluate shoulder pain, shoulder-related function, psychological distress, shoulder-related parenting disability, paid and unpaid work participation and work productivity in this patient group.

3. Explore health service utilisation and medication use for shoulder pain and associated out-of-pocket healthcare expenditure.

4. Monitor changes in well-being, work productivity and healthcare expenditure over time.

\section{METHODS AND ANALYSIS \\ Study design}

A multicentre cohort study will be undertaken. Participant recruitment commenced in May 2017, and it is
Table 1 Eligibility criteria

\begin{tabular}{ll}
\hline Inclusion criteria & Exclusion criteria \\
\hline $\begin{array}{l}\text { Shoulder pain of }>6 \text { weeks' } \\
\text { duration. }\end{array}$ & Inflammatory arthritis. \\
Aged between 20 & Same-sided fracture of \\
years and 55 years. & the scapula, humerus or \\
Fluent in English or has & clavicle within the past \\
a proxy to assist with & 12 months. \\
completion of the study & Same-sided dislocation \\
questionnaire. & of the glenohumeral joint \\
& or acromioclavicular joint \\
& within the past 3 months. \\
& Inability to provide \\
& informed consent.
\end{tabular}

*Pain that is located between the distal upper trapezius muscle and the deltoid insertion due to conditions including but not limited to: osteoarthritis, rotator cuff pathology, capsule pathology, glenohumeral instability or internal derangement of the glenohumeral joint or acromioclavicular joint.

anticipated that data collection will be completed by May 2019.

\section{Eligibility criteria}

Eligibility criteria for the study are summarised in table 1 . Individuals with shoulder pain (pain that is anatomically located between the distal upper trapezius muscle and the deltoid insertion) of more than 6 weeks' duration due to conditions including but not limited to osteoarthritis, rotator cuff pathology, capsule pathology, glenohumeral instability or internal derangement of the glenohumeral joint or acromioclavicular joint (not including acute trauma, as outlined below) will be recruited for this study. This broad range of diagnoses will enable us to capture a spectrum of relevant shoulder conditions that are associated with persistent shoulder pain.

To be eligible to participate, individuals must be aged between 20 years and 55 years and be fluent in English or have a proxy to assist with the informed consent process and completion of the study questionnaire. Exclusion criteria include inflammatory arthritis, same-sided fracture of the scapula, humerus or clavicle within the past 12 months, same-sided dislocation of the glenohumeral joint or acromioclavicular joint within the past 3 months or inability to provide informed consent. Assessment of eligibility will commence with screening of outpatient clinic lists and/or referrals and be confirmed through subsequent telephone screening by the study research assistant.

\section{Procedures for screening and recruitment}

Figure 1 presents an overview of the study procedures, including approaches for participant identification and recruitment. Participants will be recruited from the orthopaedic outpatient clinics (including specific shoulder clinics) at three major metropolitan and regional public hospitals in the state of Victoria, Australia. Clinic referrals and clinic list records at each site will be screened regularly by a senior physiotherapist to identify potentially 


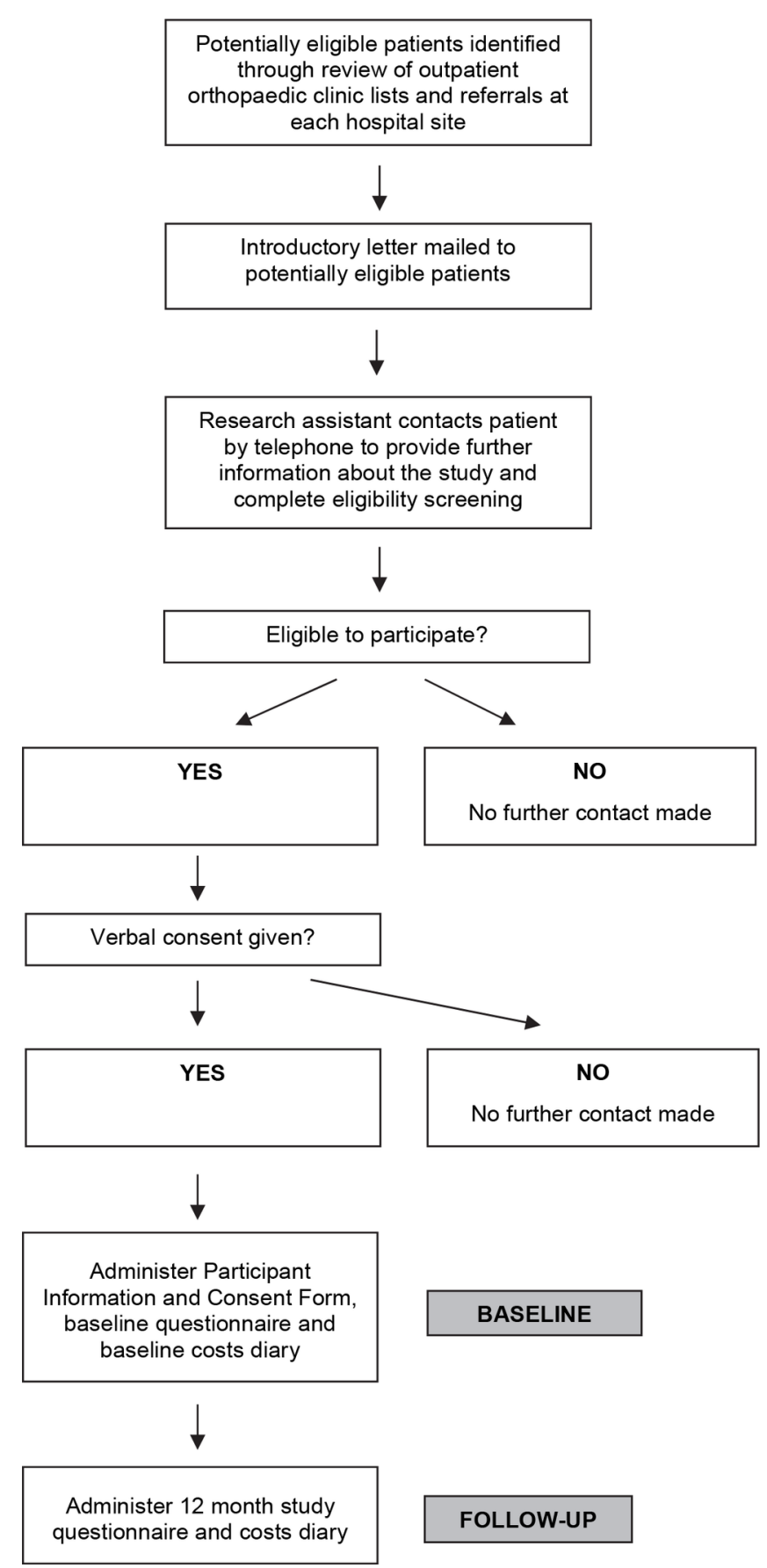

Figure 1 Overview of study procedures.

eligible individuals, based on their age and diagnosis. Completed screening forms will then be forwarded to the research team, and potentially eligible individuals will be sent an introductory letter signed by the head of orthopaedic surgery or a senior orthopaedic surgeon at that hospital site. The introductory letter will provide preliminary information about the study and invite participation in the research. After mailing of the introductory letter, the study research assistant will contact potentially eligible individuals by telephone to provide more detailed information about the study. At this time, a short screening survey to confirm eligibility will also be conducted.
Procedures for data collection

Eligible individuals who provide verbal consent will receive either an emailed participant information and consent form and individual electronic link to the electronic baseline study questionnaire or be mailed a hard copy of these documents. The electronic version of the questionnaire will contain exactly the same items as the paper-based questionnaire. For the electronic option, participants will be asked to electronically provide their consent to participate and then complete the baseline questionnaire via a secure Qualtrics online platform. For the mailed option, a reply-paid envelope will be provided to maximise response rates. The mailed option will be offered where the participant does not have an email address or is unable or unwilling to provide consent and complete the questionnaire online. All participants will be mailed a baseline costs diary for completion over a 2-week period, together with a reply-paid envelope. Non-return of mailed questionnaires and costs diaries (or missing item responses) and non-completion of online questionnaires will be followed up by telephone, mail and/or email by the research assistant.

\section{Outcome measures}

Self-reported sociodemographic data (marital status, highest level of education completed, height and weight, dominant hand and duration of shoulder pain) and information on doctor-diagnosed comorbidities (including asthma, diabetes, hypertension, increased cholesterol, coronary artery disease, anxiety or depression) will be collected as part of the study questionnaire. Participants will also be asked to specify what sources they have used to obtain information about their shoulder pain (including printed information materials, online information from websites, online pain management or education programmes, telephone helplines and social media). A range of validated plus purpose-designed patient-reported outcome measures will be administered for this study, as shown in table 2.

Table 2 Patient-reported outcome measures to be used

\section{Patient-reported outcome measure}

Key construct covered

Assessment of Quality of Life Health-related quality of life instrument

\begin{tabular}{|c|c|}
\hline Pain numerical rating scale & Shoulder pain \\
\hline Oxford Shoulder Score & Shoulder function \\
\hline QuickDASH & Shoulder function \\
\hline $\begin{array}{l}\text { Kessler Psychological } \\
\text { Distress Scale }\end{array}$ & Psychological distress \\
\hline $\begin{array}{l}\text { Modified Parenting Disability } \\
\text { Index }\end{array}$ & $\begin{array}{l}\text { Shoulder-related parenting } \\
\text { disability }\end{array}$ \\
\hline $\begin{array}{l}\text { Work Productivity and Activity } \\
\text { Impairment Questionnaire }\end{array}$ & $\begin{array}{l}\text { Shoulder-related work } \\
\text { productivity }\end{array}$ \\
\hline
\end{tabular}

*Modified from the Parenting Disability Index initially developed for rheumatoid arthritis. ${ }^{28}$ 
To achieve aim 1, HRQoL will be assessed using the generic (non-disease-specific) 12-item Assessment of Quality of Life (AQoL) instrument (AQOL-4D). ${ }^{16}$ Published Australian population norms (for the age group of 20-59 years) for the AQoL instrument, stratified by age group and sex, are available for comparison. ${ }^{17}$

To achieve aim 2, average shoulder pain over the past 7 days will be assessed using a numerical pain rating scale (with 0 indicating 'no pain' and 10 indicating 'worst pain imaginable'). Shoulder function will be evaluated using the Oxford Shoulder Score ${ }^{18}$ and the QuickDASH instrument ${ }^{19}$ (including the four-item QuickDASH work module). These instruments are widely used in shoulder dysfunction research, and their validity and reliability have been extensively demonstrated. ${ }^{20}{ }^{21}$ Both instruments were chosen for this study as they are commonly used by clinicians and researchers and also as they consider different functional activities (eg, the Oxford Shoulder Score covers difficulty with dressing and brushing or combing hair, while the QuickDASH includes difficulty with recreational activities and interference with normal social activities), thereby providing complementary information. The Kessler Psychological Distress Scale (K10) will be used to assess psychological distress. ${ }^{22}$ High K10 scores have been found to be strong predictors of depression and anxiety, ${ }^{22}$ and Australian population norms are available for comparison from the 2014-2015 National Health Survey, stratified by age group and sex. ${ }^{23}$ Information on paid and unpaid work participation (type of work, hours of work and inability to undertake paid or unpaid work due to shoulder pain) will be collected within the study questionnaire. Shoulder-related work productivity will be assessed using the Work Productivity and Activity Impairment Questionnaire (WPAI) (Specific Health Problem V.2.0), which enables the items to refer to a specific health condition (in this case 'shoulder pain'). ${ }^{24}{ }^{25}$ The Australian and New Zealand Standard Classification of Occupations (ANZSCO) V.1.2, $2013^{26}$ will be used to classify each participant's occupation and estimate lost earnings due to shoulder pain. ${ }^{27}$ The ANZSCO classification includes 8 major occupational groups, 43 submajor occupational groups and 97 minor occupational groups, allowing a high level of granularity. For female and male participants who have children, shoulder-related parenting disability will be evaluated using a modified version of the Parenting Disability Index. ${ }^{28}$ This instrument was originally developed for assessing parenting disability among people with rheumatoid arthritis; however, it has since been used in studies involving people with systemic sclerosis and systemic lupus erythematosus ${ }^{29}$ and early inflammatory arthritis. ${ }^{30}$ While measures of parenting self-efficacy and parenting stress are available, this instrument was selected for the current study given its focus on physical activities. In particular, it covers a broad range of pertinent parenting tasks that require adequate upper limb functioning, such as getting a child in and out of a car seat, using a stroller, administering medications and playing with a child. The Parenting Disability Index has a section for parents of younger children aged $0-5$ years and a section for parents of older children aged 6-18 years. For this study, each item was modified to refer to 'shoulder pain' rather than 'rheumatoid arthritis', and the response option 'did not do for reasons other than rheumatoid arthritis' was modified to refer to 'shoulder pain'. Other minor modifications to item wording were also made to reflect local terminology (eg, 'changing diapers' was reworded to 'changing nappies').

Aim 3 will be achieved by asking participants about their use of shoulder pain-related health services during the previous 12 months (for visits to orthopaedic surgeons, rheumatologists and/or sports physicians/sports doctors) and during the previous 3 months (for visits to general practitioners, physiotherapists, chiropractors, osteopaths, massage therapists/remedial masseuses/myotherapists and/or acupuncturists). Participants will also be asked whether they have had shoulder surgery or joint injections and about their use of prescribed and non-prescribed medications or supplements for shoulder pain. Out-ofpocket healthcare and medication expenditure (expenditure that is not reimbursed by government, private health insurance or other sources) will be collected for a 2-week period using a purpose-designed costs diary. This timeframe was chosen to minimise participant burden and maximise cost diary return rates. The costs diary is structured to capture expenditure on medical appointments, non-medical appointments, medications and supplements and medical tests (eg, radiographic, ultrasound, MRI and/or blood tests) specifically for shoulder pain.

To address aim 4, participants will be sent a follow-up questionnaire at 12 months from the date of completing the baseline questionnaire. A follow-up costs diary will also be mailed to all participants at 12 months for collecting out-of-pocket healthcare and medication expenditure over a 2-week period.

\section{Sample size considerations}

As the primary aim is to compare HRQoL for the study sample with population norms, sample size calculations were based on normative AQoL data from the Australian population aged $20-59$ years. ${ }^{17}$ An overall sample size of 126 is estimated to provide $80 \%$ power to detect a difference in HRQoL of 0.06 AQoL units between study participants with shoulder pain and the Australian population aged 20-59 years (assuming $\mathrm{SD}=0.24$, two-tailed test, alpha $=0.05$ ). This is considered to be a conservative estimate of difference, based on the published minimal important difference for the AQoL instrument. ${ }^{17}$ Our previous research involving people aged 20-55 years with hip or knee osteoarthritis $(\mathrm{n}=147)$ identified much larger reductions in HRQoL, compared with age-matched Australian population norms (mean reduction of 0.35 AQoL units, $95 \%$ CI 0.31 to 0.40$){ }^{1}$

As this study involves longitudinal data collection, the sample size has been increased to 150 participants (across the three hospital sites) to allow for a potential $20 \%$ dropout between baseline and 12-month follow-up. 
Our discussions with senior orthopaedic surgeons at each hospital site indicate that recruiting this sample size is feasible within the proposed timeline (eg, a review of outpatient clinic visits showed that 40 potentially eligible patients were seen in a 12-week period at one site alone). It is proposed that approximately 50 participants will be recruited from each of the three hospital sites; however, the study will not compare participants across the hospital sites, and the actual number recruited from each site may vary depending on clinical caseload, volume of outpatient clinic bookings and referrals, and eligibility factors.

\section{Planned statistical analyses}

Data analysis will be undertaken using IBM SPSS Statistics V.23. Published scoring guidelines and available algorithms will be used to generate AQoL, Oxford Shoulder Score, QuickDASH, K10, WPAI and parenting disability scores. ${ }^{228}{ }^{31-34}$ Baseline sociodemographic, employment, HRQoL, shoulder pain, shoulder function, psychological distress, work productivity and parenting disability data will be analysed descriptively. Baseline HRQoL data will be compared with Australian population norms ${ }^{17}$ (overall and stratified by age group and sex) using independent t-tests. Baseline K10 data will be categorised for analysis using published definitions of psychological distress (K10 scores $<16$ indicate low distress, 16-21 indicate moderate distress, 22-29 indicate high distress and $\geq 30$ represent very high distress) ${ }^{23}$ and compared with Australian population norms ${ }^{23}$ using the relative risk statistic.

Associations between sociodemographic factors, shoulder pain, shoulder function, HRQoL, psychological distress and work productivity will be evaluated using univariate and multivariate analyses. Data on paid and unpaid work participation, health service utilisation, medication use and out-of-pocket healthcare expenditure will be analysed descriptively. Recent Australian Bureau of Statistics labour force data on average weekly and hourly earnings for ANZSCO occupation categories ${ }^{27}$ will be used to estimate the financial cost of reduced work participation due to shoulder pain. Changes in HRQoL, shoulder pain, shoulder function, psychological distress, work productivity and parenting scores from baseline to 12 months will be analysed using paired t-tests. Any changes in out-of-pocket healthcare expenditure will be analysed using paired t-tests or non-parametric Wilcoxon signedrank tests. A subgroup analysis may also be performed for participants who undergo shoulder surgery during the follow-up period.

\section{Patient and public involvement}

Patients were not directly involved in the development of the research question; however, the study design and selection of outcome measures were informed by our earlier research involving younger people with osteoarthritis.

\section{DISCUSSION}

Little is known about the well-being or work limitations experienced by younger adults with shoulder pain or health service utilisation by this patient group. In particular, comprehensive data on the healthcare costs incurred (in relation to general practitioner, medical specialist, surgeon and allied health consultations, as well as diagnostic tests and prescribed and non-prescribed medications including analgesic and anti-inflammatory drugs) are not available. Using an efficient methodology, this study will generate comprehensive information about the burden of shoulder pain among younger people presenting to metropolitan and regional public hospital outpatient services. With broad eligibility criteria, the study has been designed to capture a range of painful shoulder conditions affecting people of working age, although the specific diagnoses may vary by age within the cohort (eg, internal derangement may be more common among participants aged 20-30 years and rotator cuff pathology and osteoarthritis may be more common towards the upper age limit of 55 years). We intend to report the type and frequency of shoulder diagnoses in order to fully characterise the study sample.

Improving our understanding of the personal, work-related and financial impacts of painful shoulder conditions is essential for optimising patient care and planning future health service delivery. The longitudinal study design will also enable us to track the burden of shoulder pain over a 12-month period. Our earlier research involving other patient groups with persistent musculoskeletal pain (including patients on waiting lists for joint replacement surgery and younger patients with hip or knee osteoarthritis) has revealed marked reductions in quality of life and high levels of distress, compared with population norms. ${ }^{135}$ These findings have contributed to the development of innovative new models of care, such as the Osteoarthritis Hip and Knee Service that has improved patient access to non-surgical management and orthopaedic surgery, and new approaches for screening patients for psychological distress in hospital orthopaedic outpatient settings. The research team also has expertise in examining the socioeconomic determinants of musculoskeletal healthcare, ${ }^{36} 37$ and this study provides new opportunities for identifying and addressing inequities in health service utilisation among patients accessing the public healthcare system.

\section{Limitations}

Although all recruitment and data collection will be undertaken within one Australian state, we anticipate that our multisite recruitment strategy (comprising a large metropolitan tertiary public hospital, a smaller metropolitan tertiary public hospital and a regional tertiary public hospital, each with a sizeable catchment area) will enable the findings to be generalised more broadly to younger Australians with persistent shoulder pain. However, we acknowledge that there are likely to be differences in waiting times, healthcare costs and socioeconomic status (including employment and capacity to pay for healthcare) for patients accessing the public hospital system, compared with those who are privately 
insured, and that the findings may not be generalisable to patients accessing private orthopaedic services. This study focuses on orthopaedic outpatient clinic settings, and we recognise that patients seen only in primary care settings will not be captured. However, in Australia, patients who have ongoing shoulder pain would be referred for specialist opinion and management. We also recognise that it would be valuable to maintain the cohort and track longer term trajectories in well-being and work participation in relation to shoulder pain, but this is reliant on obtaining additional research funding. Finally, this study will collect important information on personal out-ofpocket healthcare costs but will not examine costs from a healthcare system perspective.

\section{Ethics and dissemination}

This study will be carried out according to the Australian National Statement on Ethical Conduct in Human Research. ${ }^{38}$ Informed consent will be obtained from all study participants, and participants are free to withdraw from the study at any time. Given the study's non-interventional nature, we do not anticipate any risks to individuals as a result of their participation in this research and access to care will not be affected by participation or non-participation. All data will be stored securely at the coordinating site (Department of Epidemiology and Preventive Medicine, Monash University) and will only be accessible to authorised study staff. The data will be reidentifiable, with a unique code assigned to each participant for use on the questionnaires and costs diaries. Name and contact information will be stored separately to any information provided as part of the study questionnaires and costs diaries.

The study findings will be reported according to the Strengthening the Reporting of Observational Studies in Epidemiology checklist ${ }^{39}$ and submitted to peer-reviewed journals for publication. They will also be presented at relevant national and international scientific meetings. It is anticipated that the results will be submitted to musculoskeletal consumer organisations for broader dissemination, potentially via their website, member communications and/or policy documents. A detailed summary of the results will also be submitted to the funding body to fulfil grant reporting requirements.

\section{Author affiliations}

${ }^{1}$ Department of Epidemiology and Preventive Medicine, Monash University Faculty of Medicine Nursing and Health Sciences, Melbourne, Victoria, Australia

${ }^{2}$ Barwon Orthopaedic Research and Education, Barwon Health and School of Medicine, Deakin University, Geelong, Victoria, Australia

${ }^{3}$ Physiotherapy Department, Barwon Health, Geelong, Victoria, Australia

${ }^{4}$ Orthopaedic Department, Frankston Hospital, Frankston, Victoria, Australia

${ }^{5}$ Department of Surgery, Central Clinical School, Monash University, Melbourne, Victoria, Australia

${ }^{6}$ Department of Medicine - Western Health, The University of Melbourne,

Melbourne, Victoria, Australia

${ }^{7}$ Australian Institute for Musculoskeletal Science, Melbourne, Victoria, Australia

${ }^{8}$ Department of Orthopaedic Surgery, The Royal Melbourne Hospital, Melbourne, Victoria, Australia
${ }^{9}$ Physiotherapy Department, The Royal Melbourne Hospital, Melbourne, Victoria, Australia

Acknowledgements The authors wish to thank Mr Mark Tacey (Monash University) for his assistance with sample size calculations. We are also grateful to Ms Sally Beattie (Barwon Health), Dr Cylie Williams (Peninsula Health), Dr Vicky Tobin (Peninsula Health), Ms Nicola Bunting-Frame (Peninsula Health) and Ms Bernarda Cavka (Melbourne Health) for their assistance at the hospital sites.

Contributors INA and RSP conceived this work and secured research funding. All authors contributed to the development and refinement of the study protocol. All authors contributed to manuscript preparation. All authors have approved the final version of the manuscript.

Funding This study is supported by a research grant from the Victorian Orthopaedic Research Trust and in-kind support from Monash University, with additional support from a targeted donation via the Barwon Centre for Orthopaedic Research and Education. Dr Sharon Brennan-OIsen is supported by a National Health and Medical Research Council of Australia Career Development Fellowship (\#1107510).

Competing interests Professor RSP has received institutional educational support from De Puy-Synthes and research investigator support from Integra Health. Dr Brennan-Olsen has received personal fees from Amgen Australia, outside the submitted work.

Patient consent Not required.

Ethics approval Ethics approval has been obtained from the Barwon Health and Melbourne Health Human Research Ethics Committees, and the study has been registered with the Monash University Human Research Ethics Committee.

Provenance and peer review Not commissioned; externally peer reviewed.

Open access This is an open access article distributed in accordance with the Creative Commons Attribution Non Commercial (CC BY-NC 4.0) license, which permits others to distribute, remix, adapt, build upon this work non-commercially, and license their derivative works on different terms, provided the original work is properly cited, appropriate credit is given, any changes made indicated, and the use is non-commercial. See: http://creativecommons.org/licenses/by-nc/4.0/.

\section{REFERENCES}

1. Ackerman IN, Bucknill A, Page RS, et al. The substantial personal burden experienced by younger people with hip or knee osteoarthritis. Osteoarthritis Cartilage 2015;23:1276-84.

2. Hill CL, Gill TK, Shanahan EM, et al. Prevalence and correlates of shoulder pain and stiffness in a population-based study: the North West Adelaide Health Study. Int J Rheum Dis 2010;13:215-22.

3. Luime JJ, Koes BW, Hendriksen IJ, et al. Prevalence and incidence of shoulder pain in the general population; a systematic review. Scand J Rheumatol 2004;33:73-81.

4. van der Windt DA, Thomas E, Pope DP, et al. Occupational risk factors for shoulder pain: a systematic review. Occup Environ Med 2000;57:433-42.

5. Ostergren PO, Hanson BS, Balogh I, et al. Incidence of shoulder and neck pain in a working population: effect modification between mechanical and psychosocial exposures at work? Results from a one year follow up of the Malmö shoulder and neck study cohort. $J$ Epidemiol Community Health 2005;59:721-8.

6. Walker H, Gabbe B, Wajswelner H, et al. Shoulder pain in swimmers: a 12-month prospective cohort study of incidence and risk factors. Phys Ther Sport 2012;13:243-9.

7. Menge TJ, Byram IR, Boykin RE, et al. Labrum and rotator cuff injuries in the throwing athlete. Phys Sportsmed 2015;43:65-72.

8. McRae S, Leiter J, Walmsley C, et al. Relationship between selfreported shoulder function/quality of life, body mass index, and other contributing factors in patients awaiting rotator cuff repair surgery. $J$ Shoulder Elbow Surg 2011;20:57-61.

9. Piitulainen $\mathrm{K}$, Ylinen J, Kautiainen $\mathrm{H}$, et al. The relationship between functional disability and health-related quality of life in patients with a rotator cuff tear. Disabil Rehabil 2012;34:2071-5.

10. Cho $\mathrm{CH}$, Seo HJ, Bae KC, et al. The impact of depression and anxiety on self-assessed pain, disability, and quality of life in patients scheduled for rotator cuff repair. J Shoulder Elbow Surg 2013;22:1160-6.

11. Bilberg R, Nørgaard B, Overgaard S, et al. Mental health and quality of life in shoulder pain patients and hip pain patients assessed by patient reported outcome. Int J Orthop Trauma Nurs 2014;18:81-8. 
12. Fernandes MR. Correlation between functional disability and quality of life in patients with adhesive capsulitis. Acta Ortop Bras 2015;23:81-4.

13. Gartsman GM, Brinker MR, Khan M, et al. Self-assessment of general health status in patients with five common shoulder conditions. J Shoulder Elbow Surg 1998;7:228-37.

14. Nyman T, Grooten WJ, Wiktorin C, et al. Sickness absence and concurrent low back and neck-shoulder pain: results from the MUSIC-Norrtälje study. Eur Spine J 2007;16:631-8.

15. van den Heuvel SG, ljmker S, Blatter BM, et al. Loss of productivity due to neck/shoulder symptoms and hand/arm symptoms: results from the PROMO-study. J Occup Rehabil 2007;17:370-82.

16. Hawthorne G, Richardson J, Day N. Using the Assessment of Quality of Life (AQoL) Version 1. Melbourne: Centre for Health Program Evaluation, 2001.

17. Hawthorne G, Korn S, Richardson J. Population norms for the AQoL derived from the 2007 Australian National Survey of Mental Health and Wellbeing. Aust N Z J Public Health 2013;37:7-16.

18. Dawson J, Rogers K, Fitzpatrick R, et al. The Oxford shoulder score revisited. Arch Orthop Trauma Surg 2009;129:119-23.

19. Kennedy C, Beaton D, Solway S, et al. Disabilities of the Arm, Shoulder and Hand (DASH). The DASH and QuickDASH Outcome Measure User's Manual. 3rd edn. Toronto: Institute for Work and Health, 2011.

20. Angst F, Schwyzer H-K, Aeschlimann A, et al. Measures of adult shoulder function: Disabilities of the Arm, Shoulder, and Hand Questionnaire (DASH) and Its Short Version (QuickDASH), Shoulder Pain and Disability Index (SPADI), American Shoulder and Elbow Surgeons (ASES) Society Standardized Shoulder Assessment Form, Constant (Murley) Score (CS), Simple Shoulder Test (SST), Oxford Shoulder Score (OSS), Shoulder Disability Questionnaire (SDQ), and Western Ontario Shoulder Instability Index (WOSI). Arthritis Care Res 2011;63:S174-88.

21. Kennedy CA, Beaton DE, Smith $\mathrm{P}$, et al. Measurement properties of the QuickDASH (disabilities of the arm, shoulder and hand) outcome measure and cross-cultural adaptations of the QuickDASH: a systematic review. Qual Life Res 2013;22:2509-47.

22. Andrews $G$, Slade T. Interpreting scores on the Kessler Psychological Distress Scale (K10). Aust N Z J Public Health 2001;25:494-7.

23. Australian Bureau of Statistics. 4364.0.55.001 - National Health Survey: First results 2014-15. http://www.abs.gov.au/ausstats/abs@. $\mathrm{nsf} / \mathrm{mf} / 4364.0 .55 .001$ (accessed 13 Sep 2017).

24. Reilly Associates. WPAI General Information. http://www. reillyassociates.net/WPAl_General.html (accessed 13 Sep 2017).

25. Tang K, Beaton DE, Boonen A, et al. Measures of work disability and productivity: Rheumatoid Arthritis Specific Work Productivity Survey (WPS-RA), Workplace Activity Limitations Scale (WALS), Work Instability Scale for Rheumatoid Arthritis (RA-WIS),
Work Limitations Questionnaire (WLQ), and. Arthritis Care Res 2011;63:S337-49.

26. Australian Bureau of Statistics. 1220.0 - ANZSCO - Australian and New Zealand Standard Classification of Occupations, 2013, Version 1.2. http://www.abs.gov.au/AUSSTATS/abs@.nsf/DetailsPage/1220. 02013,\%20Version\%201.2?OpenDocument (accessed 13 Sep 2017).

27. Australian Bureau of Statistics. 6306.0 - Employee Earnings and Hours, Australia, May 2016. http://www.abs.gov.au/AUSSTATS/ abs@.nsf/DetailsPage/6306.0May\%202016?OpenDocument (accessed 13 Sep 2017).

28. Katz PP, Pasch LA, Wong B. Development of an instrument to measure disability in parenting activity among women with rheumatoid arthritis. Arthritis Rheum 2003;48:935-43.

29. Poole JL, Hare KS, Turner-Montez S, et al. Mothers with chronic disease: a comparison of parenting in mothers with systemic sclerosis and systemic lupus erythematosus. OTJR 2014;34:12-19.

30. Zelkowitz P, Looper KJ, Mustafa SS, et al. Parenting disability, parenting stress and child behaviour in early inflammatory arthritis. Chronic Dis Inj Can 2013;33:81-7.

31. Monash University Centre for Health Economics. Scoring Psychometric (unweighted) or utility (weighted)? http://aqol.com.au/ index.php/scoring-algorithms (accessed 13 Sep 2017).

32. Oxford University Innovation. The Oxford Shoulder Score (OSS). https://innovation.ox.ac.uk/outcome-measures/oxford-shoulderscore-oss/ (accessed 13 Sep 2017).

33. Institute for Work and Health. About the QuickDASH. http://www. dash.iwh.on.ca/about-quickdash (accessed 13 Sep 2017).

34. Reilly Associates. WPAI Scoring. http://www.reillyassociates.net/ WPAl_Scoring.html (accessed 13 Sep 2017).

35. Ackerman IN, Graves SE, Wicks IP, et al. Severely compromised quality of life in women and those of lower socioeconomic status waiting for joint replacement surgery. Arthritis Rheum 2005;53:653-8.

36. Brennan SL, Stanford T, Wluka AE, et al. Cross-sectional analysis of association between socioeconomic status and utilization of primary total hip joint replacements 2006-7: Australian Orthopaedic Association National Joint Replacement Registry. BMC Musculoskelet Disord 2012;13:63.

37. Brennan SL, Stanford T, Wluka AE, et al. Utilisation of primary total knee joint replacements across socioeconomic status in the Barwon Statistical Division, Australia, 2006-2007: a cross-sectional study. BMJ Open 2012;2: 001310 .

38. National Health and Medical Research Council. National statement on ethical conduct in human research (2007) incorporating all updates as at May 2015. https://www.nhmrc.gov.au/guidelinespublications/e72 (accessed 13 Sep 2017).

39. von Elm E, Altman DG, Egger M, et al. Strengthening the Reporting of Observational Studies in Epidemiology (STROBE) statement: guidelines for reporting observational studies. BMJ 2007;335:806-8. 